\title{
Medical Image of the Week: Malposition of Central Venous Catheter
}

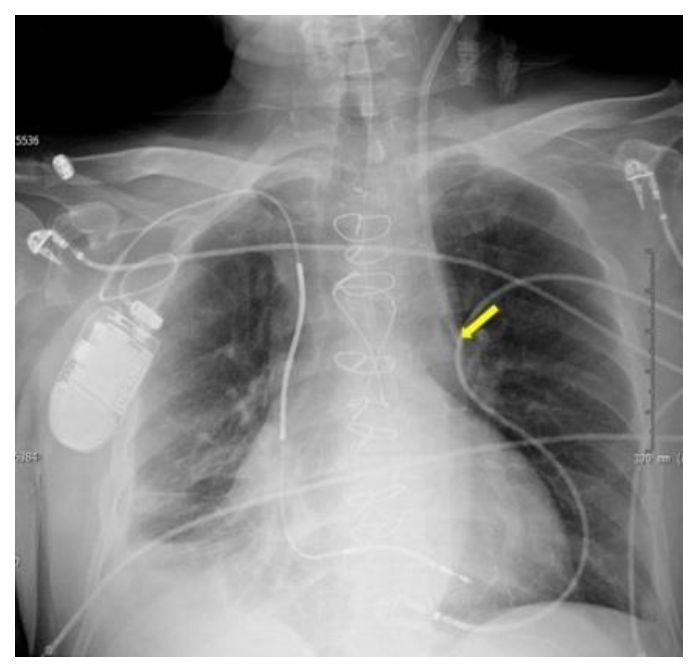

Figure 1. Portable anterior-posterior chest $x$-ray showing the tip of the catheter projecting on the left lung filed instead of crossing the midline.

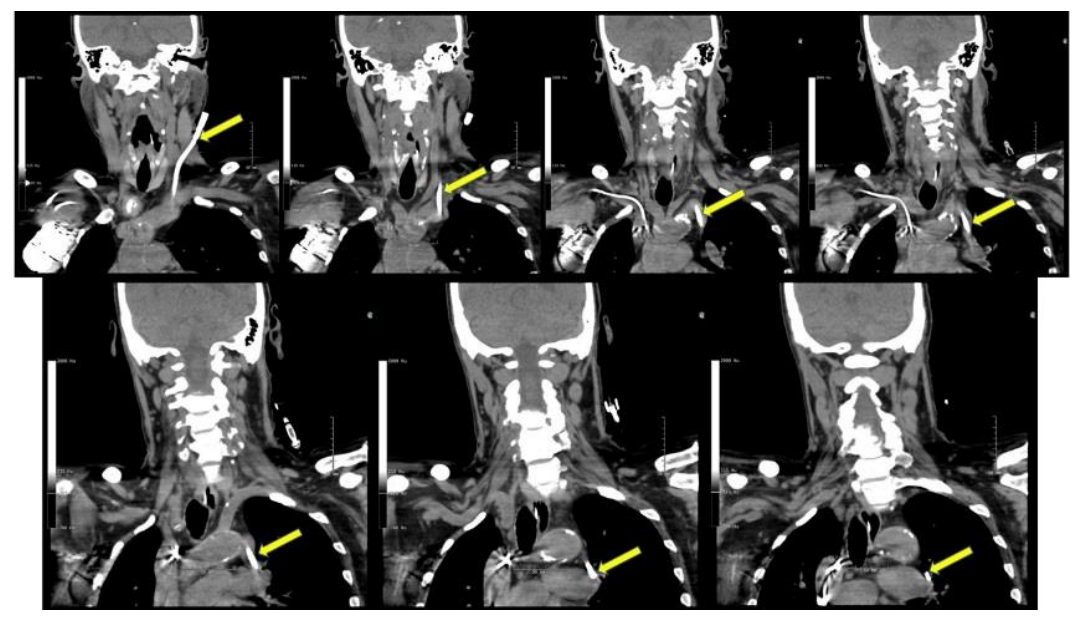

Figure 2. Coronal images of computed tomography of head, neck, and upper chest. Yellow arrows showing the anatomical course of the left internal jugular catheter. Left upper image showing the catheter entering the internal jugular vein. Right lower image showing the tip of the catheter in the left inferior pulmonary vein.

A 66-year-old man a with history of systolic heart failure and end-stage renal disease on hemodialysis was admitted to the intensive care unit due to cardiogenic shock requiring inotropes. As left arm fistula was clotted, left internal jugular vein triple-lumen catheter (IJC) was placed to obtain a hemodialysis access. Central line placement was performed under ultrasound guidance with no complications. A confirmatory chest x-ray revealed central venous catheter malposition; the catheter tip did not cross the midline; 
instead, it projected over the left lung field which was concerning for arterial puncture of the carotid artery (Figure 1). Bedside ultrasonography showed an appropriate catheter placement in the left internal jugular vein, but the final catheter tip location was unclear. The transduced pressure was low; approximately $5 \mathrm{mmHg}$. A blood gas sample from the catheter was compatible with arterial blood; $\mathrm{pH} 7.42, \mathrm{pCO} 234$, and $\mathrm{pO} 292$. Computed tomography scan of the head and neck showed the IJC entering the left jugular vein, coursing within an anomalous left pulmonary vein, and terminating within the left inferior pulmonary vein (Figure 2). The catheter was not used and was withdrawn without complications.

One of the notable complications of central venous catheter (CVC) placement is malposition, with an approximate rate of 6,7\% (1). Catheter malposition indicates that the catheter tip lies outside the recommended position (within the mid lower part of the superior vein cava (SVC) above its junction with the right atrium and parallel to the vessel walls). Possible sites of central catheter malposition include the carotid artery, azygos vein, persistent left-sided SVC, internal mammary vein, vertebral vein, pericardium, pleural space, thoracic duct and mediastinum (2). As artery puncture in the carotid artery can lead to serious complications, malposition of the catheter should be addressed in a stepwise approach. Initially bedside ultrasound should be performed to determine the anatomical catheter course and the position of the tip. A pressure transducer is also helpful in differentiating venous versus arterial waveform and measuring the transduced pressure, obtaining arterial blood gases and eventually confirming the catheter position with CT scan or CT angiography. Malposition of the jugular catheterization incidentally revealing partial anomalous of pulmonary venous return was described in a very few cases in literature, the catheter was used for seven days for continuous veno-venous hemofiltration in one of these cases (3). At this time there is insufficient literature to determine the safety of using CVC inserted in an anomalous pulmonary vein.

Mohamad Muhailan, MD and Muhamad Alhaj Moustafa, MD Department of Internal Medicine MedStar Washington Hospital Center, Internal Medicine Washington, DC, USA

\section{References}

1. Schummer W, Schummer C, Rose N, Niesen WD, Sakka SG. Mechanical complications and malposition of central venous cannulations by experienced operators. A prospective study of 1794 catheterizations in critically ill patients. Intensive Care Med. 2007 Jun;33(6):1055-9. [CrossRef] [PubMed]

2. Wang L, Liu ZS, Wang CA. Malposition of central venous catheter: Presentation and management. Chin Med J (Engl). 2016 Jan 20;129(2):227-34. [CrossRef] [PubMed]

3. Grillot N, Figueiredo S, Aubry A, Leblanc PE, Duranteau J. Unusual dialysis catheter position due to partial anomalous pulmonary venous return: Diagnosis and management. Anaesth Crit Care Pain Med. 2016 Jun;35(3):233-5. [CrossRef] [PubMed] 